\title{
Calculating Invariants as Coreflexive Bisimulations
}

\author{
Luís S. Barbosa ${ }^{1}$, José N. Oliveira ${ }^{1}$, and Alexandra Silva ${ }^{2, \star}$ \\ ${ }^{1}$ CCTC, Universidade do Minho, 4700-320 Braga, Portugal \\ ${ }^{2}$ Centrum voor Wiskunde en Informatica (CWI), Kruislaan 413, NL-1098 SJ Amsterdam
}

\begin{abstract}
Invariants, bisimulations and assertions are the main ingredients of coalgebra theory applied to software systems. In this paper we reduce the first to a particular case of the second and show how both together pave the way to a theory of coalgebras which regards invariant predicates as types. An outcome of such a theory is a calculus of invariants' proof obligation discharge, a fragment of which is presented in the paper.

The approach has two main ingredients: one is that of adopting relations as "first class citizens" in a pointfree reasoning style; the other lies on a synergy found between a relational construct, Reynolds' relation on functions involved in the abstraction theorem on parametric polymorphism and the coalgebraic account of bisimulations and invariants. This leads to an elegant proof of the equivalence between two different definitions of bisimulation found in coalgebra literature (due to B. Jacobs and Aczel \& Mendler, respectively) and to their instantiation to the classical Park-Milner definition popular in process algebra.
\end{abstract}

Keywords: coalgebraic reasoning; proof obligations; pointfree transform; program calculation.

\section{Introduction}

The most widespread application of computer systems today is to support business operations. For this reason, the onus is on the software developer to ensure that business rules are properly taken into account. Computer scientists regard such rules as examples of invariant properties. The word "invariant" captures the idea that such desirable properties are to be maintained invariant, that is, unharmed across all transactions which are embodied in the system's functionality.

Changing business rules (ie. invariants) has a price: the code needs to be upgraded so as to ensure that changes are properly taken into account. This calls for a general theory of invariant preservation upon which one could base such an extended static checking mechanism. And this theory requires a broad view of computer systems able to take into account data persistence and continued interaction.

Coalgebra theory, widely acknowledged as the mathematics of state-based systems [22], provides an adequate modeling framework for such systems. The basic insight in coalgebraic modelling is that of representing state-based systems by functions of type

$$
p: X \longrightarrow \mathrm{F} X
$$

\footnotetext{
* Partially supported by the Fundação para a Ciência e a Tecnologia, Portugal, under grant number SFRH/BD/27482/2006.
} 
which, for every state $x \in X$, describe the observable effects of an elementary step in the evolution of the system (i.e., a state transition). The possible outcomes of such steps are captured by notation $\mathrm{F} X$, where functor $\mathrm{F}$ acts as a shape for the system's interface.

Jacobs [11] identifies three cornerstones in the theory of coalgebras: invariants, bisimilarity and assertions. The latter are modal properties of states. About the first he writes: an important aspect of formally establishing the safety of systems is to prove that certain crucial predicates are actually invariants.

In this paper we develop a theory of invariant preservation whose novelty resides in explicitly expressing invariants as bisimulations. (See section 3 and its follow up.) The third cornerstone, assertions, is addressed in section 6 Altogether, we adopt a calculational style which stems from the explicit use of relational techniques, a body of knowledge often referred to as the algebra of programming [7].

Our starting point is Jacobs definition of an invariant for a given coalgebra [11]:

Definition 1. Let $\mathrm{F}:$ Sets $\rightarrow$ Sets be a polynomial functor. An invariant for a coalgebra c $: X \rightarrow \mathrm{F}(X)$ is a predicate $P \subseteq X$ satisfying for all $x \in X$,

$$
x \in P \Rightarrow c(x) \in \operatorname{Pred}(\mathrm{F})(P) .
$$

Pred $(\mathrm{F})(P)$ stands for the lifting of predicate $P$ via functor $\mathrm{F}$. (We will spell out the meaning of this construct very soon.)

Our approach will be to reason about (2) via the PF-transform [2]7]17] - a transformation of first order predicate formulæ into pointfree binary relation formulæ which will enable us to blend the concept of invariant with that of bisimulation in a handy way. (In fact, we will show the former is a particular case of the latter.) By pointfree we mean formulæ which are free of quantifiers and variables (points) such as $x$ above 1 .

Structure of the paper. The paper starts by PF-transforming definition (2), in section 2 to conclude that invariants are a special case of bisimulations (section 3). Section 4 recasts bisimulations in terms of Reynolds' arrow combinator and resorts to its calculational power to provide elegant proofs of equivalence between three most common definitions of bisimulation. The development of (a fragment of) the theory of invariants is pursued in section 5 upon a category of "predicates as types". Moving on, section 6 illustrates how the approach proposed in this paper can be also of use to reason about modal assertions over coalgebras. Finally, section 7 concludes and gives pointers to related and future work.

\section{Invariants PF-Transformed}

Our first step is to convert definition (2) into a binary relational formula. The principle is that of PF-transforming universally quantified formulæ by applying, from right to

\footnotetext{
${ }^{1}$ The idea of encoding predicates in terms of relations was initiated by De Morgan in the 1860s and followed by Peirce who, in the 1870s, found interesting equational laws of the calculus of binary relations [19]. The pointfree nature of the notation which emerged from this embryonic work was later further exploited by Tarski and his students [23]. In the 1980's, Freyd and Ščedrov [9] developed the notion of an allegory (a category whose morphisms are partially ordered) which finally accommodates the binary relation calculus [7] as special case.
} 
left, the definition of relational inclusion which follows,

$$
R \subseteq S \equiv\langle\forall y, x:: y R x \Rightarrow y S x\rangle
$$

for $R, S$ two binary relations 2 . In the case of (2), this means that $R$ will have to capture set (predicate) $P$ and $S$ will have to do the same for set $\operatorname{Pred}(\mathrm{F})(P)$. One of the standard ways of encoding a set $X$ as a binary relation is as follows: one defines a relation $\Phi_{X}$ such that

$$
y \Phi_{X} x \equiv y=x \wedge x \in X
$$

Relations of this kind are referred to as coreflexives because they are fragments of the identity relation $i d$ : $\Phi_{X} \subseteq i d$. For instance, set $\{1,2,3\}$ is captured by relation $\Phi_{\{1,2,3\}}=\{(1,1),(2,2),(3,3)\}$. We also need the binary relation composition operator

$$
b(R \cdot S) c \equiv\langle\exists a:: b R a \wedge a S c\rangle
$$

(read $R \cdot S$ as " $R$ after $S ")$ and to assert a rule which will prove convenient,

$$
(f b) R(g a) \equiv b\left(f^{\circ} \cdot R \cdot g\right) a
$$

where $f$ and $g$ are functions and _ ${ }^{\circ}$ denotes the relational converse operator defined by:

$$
a\left(R^{\circ}\right) b \equiv b R a
$$

In this context, we reason:

$$
\begin{aligned}
& \langle\forall x:: x \in P \Rightarrow c(x) \in \operatorname{Pred}(\mathrm{F})(P)\rangle \\
\equiv & \quad\{\forall \text {-one point rule }\} \\
& \langle\forall y, x: y=x: x \in P \Rightarrow c(y)=c(x) \wedge c(x) \in \operatorname{Pred}(\mathrm{F})(P)\rangle \\
\equiv & \quad\{\forall \text {-trading }\} \\
& \langle\forall y, x:: y=x \wedge x \in P \Rightarrow c(y)=c(x) \wedge c(x) \in \operatorname{Pred}(\mathrm{F})(P)\rangle \\
\equiv & \quad\{\text { (4) twice }\} \\
& \left\langle\forall y, x:: y \Phi_{P} x \Rightarrow c(y) \Phi_{\operatorname{Pred}(\mathrm{F})(P)} c(x)\right\rangle \\
\equiv & \quad\{\text { rule } 6 \\
& \left.\left\langle\forall y, x:: y \Phi_{P} x \Rightarrow y\left(c^{\circ} \cdot \Phi_{\operatorname{Pred}(\mathrm{F})(P)}\right\} c\right) x\right\rangle \\
\equiv & \quad\{\operatorname{rule}[3] \\
& \Phi_{P} \subseteq c^{\circ} \cdot \Phi_{\operatorname{Pred}(\mathrm{F})(P)} \cdot c
\end{aligned}
$$

Predicate $\operatorname{Pred}(\mathrm{F})(P)$ is defined in [11] (Def. 4.1.1) by induction on the structure of polynomial $F$. This can be abbreviated by regarding $F$ as a relator [5] and representing

\footnotetext{
${ }^{2}$ By $y R x$ we mean the fact that pair $(y, x)$ belongs to $R$. (Similarly for $y S x$.)
} 
$P$ by its coreflexive $\Phi_{P}$. The concept of a relator $\mathrm{F}$ extends that of functor to relations: $\mathrm{F} A$ describes a parametric type while $\mathrm{F} R$ is a relation from $\mathrm{F} A$ to $\mathrm{F} B$ provided $R$ is a relation from $A$ to $B$. Relators are monotone and commute with composition, converse and the identity. In this context, $\operatorname{Pred}(\mathrm{F})(P)$ coincides with relation $\mathrm{F} \Phi_{P}$. Thus we resume to (8) and calculate further:

$$
\begin{aligned}
& \Phi_{P} \subseteq c^{\circ} \cdot \mathrm{F} \Phi_{P} \cdot c \\
& \equiv \quad\{\text { see } 10 \text { below }\} \\
& c \cdot \Phi_{P} \subseteq \mathrm{F} \Phi_{P} \cdot c
\end{aligned}
$$

where the last step is justified by the first of the following laws of the relational calculus,

$$
\begin{aligned}
f \cdot R \subseteq S & \equiv R \subseteq f^{\circ} \cdot S \\
R \cdot f^{\circ} \subseteq S & \equiv R \subseteq S \cdot f
\end{aligned}
$$

known as the shunting rules [7] 3 .

Altogether, we arrive at (9), a quite compact version of (2). It tells that wherever $c$ runs on states satisfying $P$, any of its successor states will do so. The sections which follow will give evidence of the advantages of such a transformation.

\section{Invariants Are Bisimulations}

We move on to the second cornerstone of coalgebra theory - bisimilarity. This is based on the concept of bisimulation which is given by Jacobs [11] as follows:

Definition 2. A bisimulation for coalgebras $c: X \rightarrow \mathrm{F}(X)$ and $d: Y \rightarrow \mathrm{F}(Y)$ is a relation $R \subseteq X \times Y$ which is closed under $c$ and $d$ :

$$
(x, y) \in R \Rightarrow(c(x), d(y)) \in \operatorname{Rel}(\mathrm{F})(R) .
$$

for all $x \in X$ and $y \in Y$.

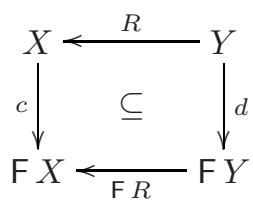

(a)

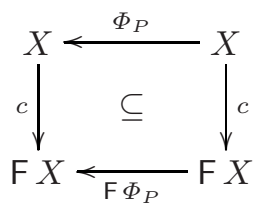

(b)

This time $\operatorname{Rel}(\mathrm{F})(R)$ stands for the relational lifting of $R$ via functor $\mathrm{F}$ which, in our relational setting, is captured by notation $\mathrm{F} R$.

An exercise at all similar to the one carried out in the previous section will show (12) PF-transformed into

$$
c \cdot R \subseteq \mathrm{F} R \cdot d
$$

as depicted in diagram (a) above, where $X$ and $Y$ are the carriers of coalgebras $c$ and $d$, respectively. Since (9) instantiates (13) we have that invariants are special cases of bisimulations: exactly those which are coreflexive relations, cf. diagram (b).

\footnotetext{
${ }^{3}$ Functions are denoted by lowercase characters (eg. $\left.f, g, \phi\right)$ and function application will be abbreviated by juxtaposition, eg. $f$ a instead of $f(a)$. Coalgebras qualify for these rules because they are functions.
} 
We shall see briefly that this conclusion brings about its benefits, as much of the theory of coalgebraic invariants stems directly from that of bisimulations 4 . We will address this one first.

\section{Calculating Bisimulations}

Let us first show how the classical definition of bisimulation used in process algebra (due to Milner and Park [18]) can be retrieved from (13) simply by instantiating $F$ to the powerset relator $\mathcal{P} X=\{S \mid S \subseteq X\}$. We need the universal property of the powertranspose isomorphism $\Lambda$

$$
f=\Lambda R \equiv R=\in \cdot f
$$

which converts binary relations to set-valued functions [7], where $A \stackrel{\epsilon}{\leftarrow} \mathcal{P} A$ is the membership relation. In [7] the powerset relator is defined by

$$
\mathcal{P} R=(\in \backslash(R \cdot \in)) \cap\left(\in \backslash\left(R^{\circ} \cdot \in\right)\right)^{\circ}
$$

where $\cap$ denotes relation intersection and $R \backslash S$ denotes relational division,

$$
a(R \backslash S) c \equiv\langle\forall b: b R a: b S c\rangle
$$

a relational operator whose semantics is captured by universal property

$$
R \cdot X \subseteq S \equiv X \subseteq R \backslash S
$$

The main ingredient of the calculation below is (14), which ensures that every powerset coalgebra uniquely determines a binary relation ( $\Lambda$ is a bijection). In this context, let $R$ be a bisimulation between two powerset coalgebras $\Lambda S$ and $\Lambda U$. We reason:

$$
\begin{aligned}
& (\Lambda S) \cdot R \subseteq(\mathcal{P} R) \cdot(\Lambda U) \\
\equiv & \quad\{\text { unfolding } \mathcal{P} R \text { [15 }\} \\
& (\Lambda S) \cdot R \subseteq(\in \backslash(R \cdot \in)) \cap\left(\in \backslash\left(R^{\circ} \cdot \in\right)\right)^{\circ} \cdot(\Lambda U) \\
\equiv & \quad\{\text { distribution (since } \Lambda U \text { is a function) thanks to } 11\}\} \\
& (\Lambda S) \cdot R \subseteq(\in \backslash(R \cdot \in)) \cdot(\Lambda U) \wedge(\Lambda S) \cdot R \subseteq\left(\in \backslash\left(R^{\circ} \cdot \in\right)\right)^{\circ} \cdot(\Lambda U) \\
\equiv & \quad\{\text { property } R \backslash(S \cdot f)=(R \backslash S) \cdot f ; \text { converses }\} \\
& (\Lambda S) \cdot R \subseteq \in \backslash(R \cdot \in \cdot \Lambda U) \wedge R^{\circ} \cdot(\Lambda S)^{\circ} \subseteq(\Lambda U)^{\circ} \cdot\left(\in \backslash\left(R^{\circ} \cdot \in\right)\right) \\
\equiv & \quad\{\text { shunting rules } \underline{10] 11} \text { and property above }\} \\
& (\Lambda S) \cdot R \subseteq \in \backslash(R \cdot \in \cdot \Lambda U) \wedge(\Lambda U) \cdot R^{\circ} \subseteq \in \backslash\left(R^{\circ} \cdot \in \cdot \Lambda S\right) \\
\equiv & \quad\{\underline{16} \text { twice }\}
\end{aligned}
$$

\footnotetext{
${ }^{4}$ It is interesting to note that Lemma 4.2.2 in [11] proves that relation $\{(x, x) \mid x \in P\}$ is a
} bisimulation yielded by invariant $P$, but no further advantage is taken from this fact. 


$$
\begin{aligned}
& \in \cdot(\Lambda S) \cdot R \subseteq R \cdot \in \cdot \Lambda U \wedge \in \cdot(\Lambda U) \cdot R^{\circ} \subseteq R^{\circ} \cdot \in \cdot \Lambda S \\
\equiv & \quad\{\text { cancellation } \in \cdot(\Lambda R)=R \text { four times }\} \\
& S \cdot R \subseteq R \cdot U \wedge U \cdot R^{\circ} \subseteq R^{\circ} \cdot S
\end{aligned}
$$

The two conjuncts state that $R$ and its converse are simulations between state transition relations $S$ and $U$, which corresponds to the Park-Milner definition 5: a bisimulation is a simulation between two LTS such that its converse is also a simulation, where a simulation between two LTS $S$ and $U$ is a relation $R$ such that, if $(p, q) \in R$, then for all $p^{\prime}$ such that $\left(p^{\prime}, p\right) \in S$, then there is a $q^{\prime}$ such that

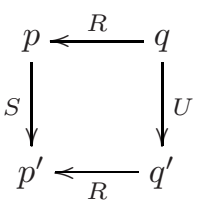
$\left(p^{\prime}, q^{\prime}\right) \in R$ and $\left(q^{\prime}, q\right) \in U$ - see diagram on the right 6 .

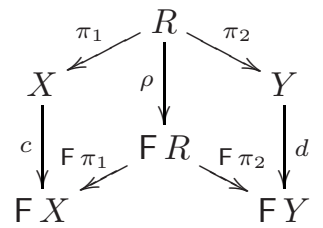

We furthermore want to check (13) against another (also coalgebraic) definition of bisimulation due to Aczel \& Mendler [1]: given two coalgebras $c: X \rightarrow F(X)$ and $d: Y \rightarrow F(Y)$ an $\mathrm{F}$-bisimulation is a relation $R \subseteq X \times Y$ which can be extended to a coalgebra $\rho$ such that projections $\pi_{1}$ and $\pi_{2}$ lift to F-coalgebra morphisms. (See diagram aside.)

Jacobs [11] spends some time in proving the equivalence between the two definitions. Our proof will be much shorter and calculational thanks to a small trick: we identify (13) as instance

$$
c(\mathrm{~F} R \leftarrow R) d
$$

of Reynolds "arrow combinator" $R \leftarrow S$ which, given $R$ and $S$, relates two functions $f$ and $g$ as follows [2]:

$$
f(R \leftarrow S) g \equiv f \cdot S \subseteq R \cdot g
$$

With points, $f(R \leftarrow S) g$ means $\langle\forall y, x:: y S x \Rightarrow(f y) R(g x)\rangle$. For instance, for $f$ and $g$ the same function and $S$ and $R$ two partial orders, 18 means that such a function is monotonic.

The fact that we can write (17) instead of $c \cdot R \subseteq \mathrm{F} R \cdot d$ (13) to mean that $R$ is a bisimulation between $\mathrm{F}$ coalgebras $c$ and $d$ is of great notational, conceptual and calculational advantage. As far as notation is concerned, 17] is very appropriate for telling that $c$ and $d$ produce $F R$-related outputs $c y$ and $d x$ provided their inputs are $R$-related ( $y \quad R x$ ). Conceptually, $\mathrm{F} R \leftarrow R$ may be regarded as a relation involving all coalgebras which are $R$-bisimilar. But it is the calculational power implicit in 17 which really justifies the recasting of (13) in terms of Reynolds' arrow combinator.

In another context, this combinator is studied in some detail in [2], where the following PF-properties can be found:

$$
i d \leftarrow i d=i d
$$

\footnotetext{
${ }^{5}$ The pointwise definition of simulation is better perceived once $S \cdot R \subseteq R \cdot U$ is re-written into $R \subseteq S \backslash(R \cdot U)$, recall [16 — similarly for the other conjunct. Matteo Vaccari [24] performs a calculation similar to the above starting directly from this pointwise definition.

${ }^{6}$ A popular presentation of the classical definition of bisimulation uses LTS defined by the labelled powerset relator $\mathcal{P}(A \times X)$, for $A$ a given set of actions. The reasoning for this functor is the same: only $\mathcal{P}(i d \times R)$ should replace $\mathcal{P} R$ in the calculation.
} 


$$
\begin{aligned}
(R \leftarrow S)^{\circ} & =R^{\circ} \leftarrow S^{\circ} \\
R \leftarrow S \subseteq V \leftarrow U & \Leftarrow R \subseteq V \wedge U \subseteq S \\
k(f \leftarrow g) h & \equiv k \cdot g=f \cdot h
\end{aligned}
$$

From property (21) we learn that the combinator is monotonic on the left hand side - and thus facts

$$
\begin{aligned}
& S \leftarrow R \subseteq(S \cup V) \leftarrow R \\
& \top \leftarrow S=\top
\end{aligned}
$$

hold 7 — and anti-monotonic on the right hand side — and thus property

$$
R \leftarrow \perp=\top
$$

and the two distributive laws which follow:

$$
\begin{aligned}
& S \leftarrow\left(R_{1} \cup R_{2}\right)=\left(S \leftarrow R_{1}\right) \cap\left(S \leftarrow R_{2}\right) \\
& \left(S_{1} \cap S_{2}\right) \leftarrow R=\left(S_{1} \leftarrow R\right) \cap\left(S_{2} \leftarrow R\right)
\end{aligned}
$$

Let us see how the properties above explain those of bisimulation by themselves. Property (20) ensures that the converse of a bisimulation is also a bisimulation. This turns out to be an equivalence:

$$
\begin{aligned}
& R \text { is a bisimulation } \\
& \equiv\{\text { 17 }\} \\
& d\left((\mathrm{~F} R)^{\circ} \leftarrow R^{\circ}\right) c \\
& c(\mathrm{~F} R \leftarrow R) d \\
& \equiv \quad\{\text { converse }\} \\
& d(\mathrm{~F} R \leftarrow R)^{\circ} c \\
& \equiv \quad\{20\} \\
& \equiv \quad\{\text { relator } \mathrm{F}\} \\
& d\left(\mathrm{~F}\left(R^{\circ}\right) \leftarrow R^{\circ}\right) c \\
& \equiv \quad\{17\} \\
& R^{\circ} \text { is a bisimulation }
\end{aligned}
$$

Next, we recall the definition of a coalgebra morphism:

Definition 3. Let $(X, p: X \longrightarrow \mathrm{F} X)$ and $(Y, q: Y \longrightarrow \mathrm{F} Y)$ be coalgebras for functor $\mathrm{F}$. A morphism connecting $p$ and $q$ is a function $h$ between their carriers such that $q \cdot h=\mathrm{F} h \cdot p$.

Clearly, property (22) tells immediately that coalgebra morphisms are bisimulations.

The easy calculation of $\mathrm{F} i d \leftarrow i d=i d$ (19) ensures $i d$ is a bisimulation between a given coalgebra and itself. On the other side of the spectrum, 25 tells us that $\perp$ is a bisimulation for any pair of coalgebras $c$ and $d$. (Just introduce points in $\mathrm{F} \perp \leftarrow \perp$ and simplify.)

Let us now see how the fact that bisimulations are closed under union,

$$
c\left(\mathrm{~F} R_{1} \leftarrow R_{1}\right) d \wedge c\left(\mathrm{~F} R_{2} \leftarrow R_{2}\right) d \Rightarrow c\left(\mathrm{~F}\left(R_{1} \cup R_{2}\right) \leftarrow\left(R_{1} \cup R_{2}\right)\right) d
$$

$\overline{{ }^{7} \text { Cf. } f \cdot S \cdot g^{\circ}} \subseteq \top \equiv$ TRUE concerning $\underline{24}$. 
stems from properties (2123) and (26). First we PF-transform (28) to

$$
\left(\mathrm{F} R_{1} \leftarrow R_{1}\right) \cap\left(\mathrm{F} R_{2} \leftarrow R_{2}\right) \subseteq \mathrm{F}\left(R_{1} \cup R_{2}\right) \leftarrow\left(R_{1} \cup R_{2}\right)
$$

and reason:

$$
\begin{aligned}
& \left(\mathrm{F} R_{1} \leftarrow R_{1}\right) \cap\left(\mathrm{F} R_{2} \leftarrow R_{2}\right) \\
& \subseteq \quad\{\text { 233 (twice) ; monotonicity of } \cap\} \\
& \left(\left(\mathrm{F} R_{1} \cup \mathrm{F} R_{2}\right) \leftarrow R_{1}\right) \cap\left(\left(\mathrm{F} R_{1} \cup \mathrm{F} R_{2}\right) \leftarrow R_{2}\right) \\
& =\{26\} \\
& \left(\mathrm{F} R_{1} \cup \mathrm{F} R_{2}\right) \leftarrow\left(R_{1} \cup R_{2}\right) \\
& \subseteq \quad\{\mathrm{F} \text { is monotonic; 21 }\} \\
& \mathrm{F}\left(R_{1} \cup R_{2}\right) \leftarrow\left(R_{1} \cup R_{2}\right)
\end{aligned}
$$

Eventually, we are in position to address the equivalence between Jacobs' and AczelMendler's definitions of bisimulation. To the set of known rules about (18) we add the following law

$$
\left(r \cdot s^{\circ}\right) \leftarrow\left(f \cdot g^{\circ}\right)=(r \leftarrow f) \cdot(s \leftarrow g)^{\circ} \Leftarrow \text { pair } r, s \text { is a tabulation }
$$

where a pair of functions $A \stackrel{r}{\longleftarrow} C \stackrel{s}{\longrightarrow} B$ form a tabulation iff split function $\langle r, s\rangle$ is injective, that is, iff $r^{\circ} \cdot r \cap s^{\circ} \cdot s=i d$ holds 8 .

Below we show that 29) is what matters in proving the equivalence between Jacobs' definition of bisimulation (once PF-transformed) and that of Aczel \& Mendler:

$$
\begin{aligned}
& c(\mathrm{~F} R \leftarrow R) d \\
\equiv \quad \quad\left\{\text { tabulate } R=\pi_{1} \cdot \pi_{2}^{\circ}\right\} & c\left(\mathrm{~F}\left(\pi_{1} \cdot \pi_{2}^{\circ}\right) \leftarrow\left(\pi_{1} \cdot \pi_{2}^{\circ}\right)\right) d \\
\equiv \quad \quad\{\text { relator commutes with composition and converse }\} & c\left(\left(\left(\mathrm{~F} \pi_{1}\right) \cdot\left(\mathrm{F} \pi_{2}\right)^{\circ}\right) \leftarrow\left(\pi_{1} \cdot \pi_{2}^{\circ}\right)\right) d \\
\equiv \quad\{\text { new rule }[29] & \text { cf. }
\end{aligned}
$$

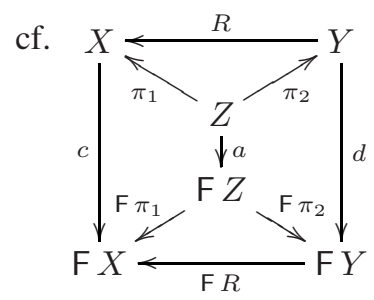

Clearly, the meaning of the last line above is exactly Aczel-Mendler's definition (cf. diagram): it states that there exists a coalgebra a whose carrier is the "graph" of bisimulation $R$ and which is such that projections $\pi_{1}$ and $\pi_{2}$ lift to the corresponding coalgebra morphisms.

\footnotetext{
${ }^{8}$ The proof of [29) can be found in [14]. It is a standard result that every $R$ can be factored in a tabulation $R=r \cdot s^{\circ}$ [7]. An obvious and easy to check tabulation is $r, s:=\pi_{1}, \pi_{2}$ [14], which boils down to pairwise equality: $(b, a)=(d, c)$ equivalent to $b=d \wedge a=c$.
} 
Note how simple the proof is. The elegance of the calculation lies in the synergy with Reynolds' arrow combinator. To the best of our knowledge, such a synergy is new in the literature 9 .

\section{Calculating Invariants}

Let us write $\mathrm{F} \Phi_{P} \longleftarrow^{c} \Phi_{P}$ to denote the fact that $P$ is an invariant (9), which we abbreviate to $\mathrm{F} \Phi \stackrel{c}{\longleftarrow} \Phi$ since predicates and coreflexives are in one to one correspondence. (We will use uppercase Greek letters to denote such coreflexives and will refer to them as "invariants" with no further explanation.)

This notation suggests a category Pred of "predicates as objects" as a suitable universe for describing coalgebraic systems subject to invariants. Pred's objects are predicates, represented by coreflexives. An arrow $\Psi \stackrel{f}{\longleftarrow} \Phi$ in Pred means a function which ensures property $\Psi$ on its output whenever property $\Phi$ holds on its input. Arrows in Pred can therefore be seen as proof-obligations for the corresponding functions 10 . Formally:

$$
\Psi \stackrel{f}{\longleftarrow} \Phi \equiv f(\Psi \leftarrow \Phi) f \equiv f \cdot \Phi \subseteq \Psi \cdot f
$$

Clearly, any relator (in Rel) restricts to a functor in Pred. In particular, the functorial image of an arrow $\Psi \stackrel{f}{\longleftarrow} \Phi$ is well-typed, cf.

$$
\begin{aligned}
& \mathrm{F} \Psi \stackrel{\mathrm{F} f}{\longleftarrow} \mathrm{F} \Phi \\
\equiv \quad & \{\underline{30}\} \\
\equiv \quad & \quad\{\text { functors }\}
\end{aligned}
$$

Such a "predicates as types" view carries over universal constructs. As Pred's hom sets are included in Set, in order to verify whether a particular universal property in

\footnotetext{
${ }^{9}$ For a longer bi-implication proof of this equivalence see Backhouse and Hoogendijk's work on final dialgebras [6]. A proof of the same result is implicit in Corollary 3.1 of [21] which invokes a result by Carboni et al [8] on extending functors to relators.

${ }^{10}$ See [16], where this view of proof obligations is actually extended to arbitrary binary relations. This is suitable for specification languages such as eg. VDM, where the inclusion of a subtyping mechanism which allows truth-valued functions forces the type checking here to rely on proofs [12].
} 
the latter lifts to a universal in Pred it is enough to check whether the corresponding diagram exists and the universal arrow in Set is still an arrow in Pred. The fact that composition satisfies constraints,

$$
\Psi \stackrel{g \cdot f}{\longleftarrow} \Phi \Leftarrow \Psi \stackrel{g}{\longleftarrow} \Upsilon \wedge \Upsilon \stackrel{f}{\longleftarrow} \Phi
$$

stems directly from 30 , as does the obvious rule concerning identity:

$$
\Psi \stackrel{i d}{\longleftarrow} \Phi \equiv \Phi \subseteq \Psi
$$

(From (31) we infer also that exponential $g^{\Phi} f=g \cdot f$ is well-typed.) For a slightly more elaborate example consider, for instance, functional products in the new setting:



Clearly, the proof-obligations associated to the two projections

$$
\pi_{1} \cdot(\Psi \times \Upsilon) \subseteq \Psi \cdot \pi_{1} \quad, \quad \pi_{2} \cdot(\Psi \times \Upsilon) \subseteq \Upsilon \cdot \pi_{2}
$$

are instances of Reynolds abstraction theorem [2025|2]:

$$
\mathrm{G} A \stackrel{f}{\longleftarrow} \mathrm{F} A \text { is polymorphic } \equiv\langle\forall R:: \quad f(\mathrm{G} R \leftarrow \mathrm{F} R) f\rangle
$$

So there is nothing to prove. To show that $\langle f, g\rangle$ is indeed an arrow in Pred we need to recall the universal property of relational splits [7]

$$
X \subseteq\langle R, S\rangle \equiv \pi_{1} \cdot X \subseteq R \wedge \pi_{2} \cdot X \subseteq S
$$

and that $\times$-absorption holds. We reason 11 :

$$
\begin{aligned}
& \Psi \times \Upsilon \stackrel{\langle f, g\rangle}{\longleftarrow} \Phi \\
\equiv & \quad\{\text { definition } 30 \text { 30 }\} \\
& \langle f, g\rangle \cdot \Phi \subseteq(\Psi \times \Upsilon) \cdot\langle f, g\rangle \\
\equiv & \quad\{\text { absorption law for relational product }\} \\
& \langle f, g\rangle \cdot \Phi \subseteq\langle\Psi \cdot f, \Upsilon \cdot g\rangle \\
\equiv & \quad\{\text { universal law for relational product 35] }\} \\
& \pi_{1} \cdot\langle f, g\rangle \cdot \Phi \subseteq \Psi \cdot f \wedge \pi_{2} \cdot\langle f, g\rangle \cdot \Phi \subseteq \Upsilon \cdot g
\end{aligned}
$$

$\overline{{ }^{11} \text { Note that the }} \Leftarrow$ part of this equivalence is also ensured by the abstraction theorem 34 of the split combinator (on functions). 


$$
\begin{aligned}
\equiv & \quad\{\text { cancellation law for functional product }\} \\
& f \cdot \Phi \subseteq \Psi \cdot f \wedge g \cdot \Phi \subseteq \Upsilon \cdot g \\
\equiv \quad & \quad \text { definition } 30 \text { twice }\} \\
& \quad \stackrel{f}{\longleftarrow} \Phi \wedge \Upsilon \stackrel{g}{\longleftarrow} \Phi
\end{aligned}
$$

As expected, a coalgebra $\mathrm{F} \Phi \stackrel{c}{\longleftarrow} \Phi$ in Pred maintains property $\Phi$ invariant. Final coalgebras (and initial algebras) exist and coincide with the ones in Set. Let us check, in this respect, the diagram of unfold (aside), where $\nu \mathrm{F}$ denotes the final coalgebra and $[(c)]$ is the coinductive extension, or unfold, of coalgebra $c$. We reason:

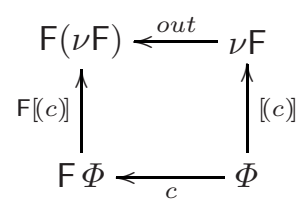

$$
\begin{aligned}
& \nu \mathrm{F} \stackrel{[(c)]}{\longleftarrow} \Phi \\
& \equiv \quad\{\text { definition } 30\} \\
& {[(c)] \cdot \Phi \subseteq[(c)]} \\
& \Leftarrow \quad\{\text { fusion: }[(T)] \cdot S \subseteq[(R)] \Leftarrow T \cdot S \subseteq \mathrm{F} S \cdot R\} \\
& c \cdot \Phi \subseteq \mathrm{F} \Phi \cdot c \\
& \equiv \quad\{\text { definition } 30\} \\
& \mathrm{F} \Phi \stackrel{c}{\longleftarrow} \Phi
\end{aligned}
$$

We close this section by showing how the "invariants as bisimulations" approach helps in developing of a number of simple, yet powerful rules to reason about "invarianttyped" coalgebras. Our calculations below address three such rules.

Separation rule:

$$
\mathrm{F}(\Phi \cdot \Psi) \stackrel{c}{\longleftarrow} \Phi \cdot \Psi \Leftarrow \mathrm{F} \Phi \stackrel{c}{\longleftarrow} \Phi \wedge \mathrm{F} \Psi \stackrel{c}{\longleftarrow} \Psi
$$

This rule enables the decomposition of the proof obligation of a compound invariant into two separate proof obligations, one per conjunct. Its calculation is as follows:

$$
\begin{aligned}
& \mathrm{F} \Phi \stackrel{c}{\longleftarrow} \Phi \wedge \mathrm{F} \Psi \stackrel{c}{ } \Psi \\
\equiv \quad & \quad\{\underline{30} \text { twice }\} \\
& c \cdot \Phi \subseteq \mathrm{F} \Phi \cdot c \wedge c \cdot \Psi \subseteq \mathrm{F} \Psi \cdot c \\
\Rightarrow \quad & \quad\{\text { monotonicity of composition (twice) }\} \\
& c \cdot \Phi \cdot \Psi \subseteq \mathrm{F} \Phi \cdot c \cdot \Psi \wedge \mathrm{F} \Phi \cdot c \cdot \Psi \subseteq \mathrm{F} \Phi \cdot \mathrm{F} \Psi \cdot c
\end{aligned}
$$




$$
\begin{aligned}
\Rightarrow & \{\text { transitivity }\} \\
& c \cdot \Phi \cdot \Psi \subseteq \mathrm{F} \Phi \cdot(\mathrm{F} \Psi \cdot c) \\
\equiv \quad & \quad\{\text { relator } \mathrm{F} \text { and }(30)\} \\
& \mathrm{F}(\Phi \cdot \Psi) \stackrel{c}{\longleftarrow} \Phi \cdot \Psi
\end{aligned}
$$

Interleaving rule:

$$
\mathrm{F}(\Phi \times \Psi) \stackrel{c\|\| d}{\longleftarrow} \Phi \times \Psi \Leftarrow \mathrm{F} \Phi \stackrel{c}{\longleftarrow} \Phi \wedge \mathrm{F} \Psi \stackrel{d}{\longleftarrow} \Psi
$$

where $\| \mid$ is an interleaving operator defined by $c\|\| d \stackrel{\text { def }}{=} \delta \cdot(c \times d)$ whenever $\mathrm{F}$ has a distributive law $\delta: \mathrm{F} \Phi \times \mathrm{F} \Psi \longrightarrow \mathrm{F}(\Phi \times \Psi)$ corresponding to the Kleisli composition of F's left and right strength (see [13] for details). The calculation of (37) follows:

$$
\begin{aligned}
& \mathrm{F} \Phi \stackrel{c}{\longleftarrow} \Phi \wedge \mathrm{F} \Psi \stackrel{d}{\longleftarrow} \Psi \\
& \equiv \quad\{30 \text { twice }\} \\
& c \cdot \Phi \subseteq \mathrm{F} \Phi \cdot c \wedge d \cdot \Psi \subseteq \mathrm{F} \Psi \cdot d \\
& \Rightarrow \quad\{\text { monotonicity of product and composition }\} \\
& \delta \cdot(c \cdot \Phi \times d \cdot \Psi) \subseteq \delta \cdot(\mathrm{F} \Phi \cdot c \times \mathrm{F} \Psi \cdot d) \\
& \Rightarrow \quad\{\times \text { relator }\} \\
& \delta \cdot(c \times d) \cdot(\Phi \times \Psi) \subseteq \delta \cdot(\mathrm{F} \Phi \times \mathrm{F} \Psi) \cdot(c \times d) \\
& \Rightarrow \quad\{\delta \text { 's free theorem } 34\} \\
& \delta \cdot(c \times d) \cdot(\Phi \times \Psi) \subseteq \mathrm{F}(\Phi \times \Psi) \cdot \delta \cdot(c \times d) \\
& \equiv \quad\{\text { definition of } c \mid \| d \text { and 30 }\} \\
& \mathrm{F}(\Phi \times \Psi) \stackrel{c\|\| d}{\longleftarrow} \Phi \times \Psi
\end{aligned}
$$

Pipeline. For F a monad,

$$
\mathrm{F} \Phi \stackrel{c \bullet d}{\longleftarrow} \Phi \Leftarrow \mathrm{F} \Phi \longleftarrow c
$$

where $c \bullet d$ corresponds to the Kleisli composition of $c$ and $d$. We calculate:

$$
\begin{aligned}
& (c \bullet d) \cdot \Phi \\
= & \quad\{\text { definition of Kleisli composition }\} \\
& \mu \cdot \mathrm{F} c \cdot d \cdot \Phi \\
\subseteq & \quad\{\mathrm{F} \Phi \stackrel{d}{\longleftarrow} \Phi \text { and monotonicity }\}
\end{aligned}
$$




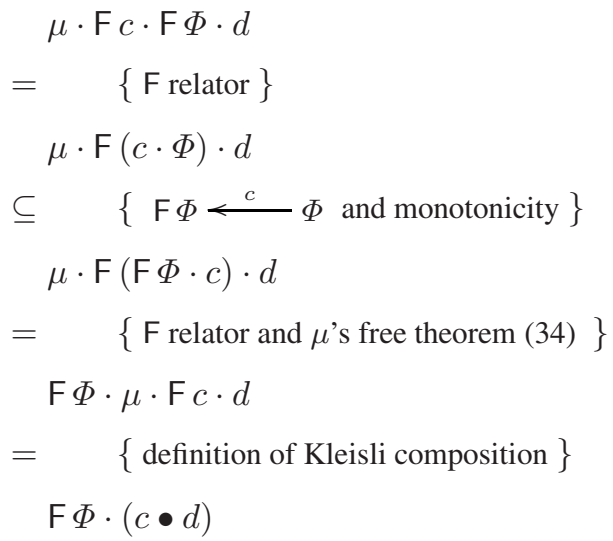

\section{Calculating Assertions}

As mentioned in the introduction to this paper, the third main ingredient of coalgebraic reasoning identified in [11] is a language of modal assertions in which specifications of the behaviour of systems can be expressed. Clearly, invariants bring about a "next time" modal operator,

$$
\begin{aligned}
c(\mathrm{~F} \Phi \leftarrow \Phi) c \equiv & c \cdot \Phi \subseteq \mathrm{F} \Phi \cdot c \\
\equiv & \{\text { shunting } 10\} \\
& \Phi \subseteq \underbrace{c \circ \cdot(\mathrm{F} \Phi) \cdot c}_{\bigcirc_{c} \Phi}
\end{aligned}
$$

which holds for those states whose all immediate successors, if any, satisfy $\Phi$. From this a PF-definition of the "next time $\Phi$ holds" modal operator emerges

$$
\bigcirc_{c} \Phi \stackrel{\text { def }}{=} c^{\circ} \cdot(\mathrm{F} \Phi) \cdot c
$$

which PF-transforms Def. 4.3.1 of [11]. So, assertion $\Phi \subseteq \bigcirc_{c} \Phi$ is an alternative statement of " $\Phi$ in an invariant" for coalgebra $c$.

This modal operator is easily shown to be the upper adjoint of Galois connection

$$
\pi_{c} \Phi \subseteq \Psi \equiv \Phi \subseteq \bigcirc_{c} \Psi
$$

whose lower adjoint is the projection operator $\pi_{c} \Phi \stackrel{\text { def }}{=} c \cdot \Phi \cdot c^{\circ}$ which is central to [15] in studying the PF-refactoring of data dependency theory (a part of database theory). From this, one immediately infers that $\bigcirc_{c}$ is monotonic and distributes over conjunction: $\bigcirc_{c}(\Phi \cdot \Psi)=\left(\bigcirc_{c} \Phi\right) \cdot\left(\bigcirc_{c} \Psi\right)$. Note that we express conjunction by composition because these two operators coincide on coreflexives:

$$
\Phi \cap \Psi=\Phi \cdot \Psi
$$


Such properties can then be used to reason about operator $\bigcirc_{c}$, as in, for example,

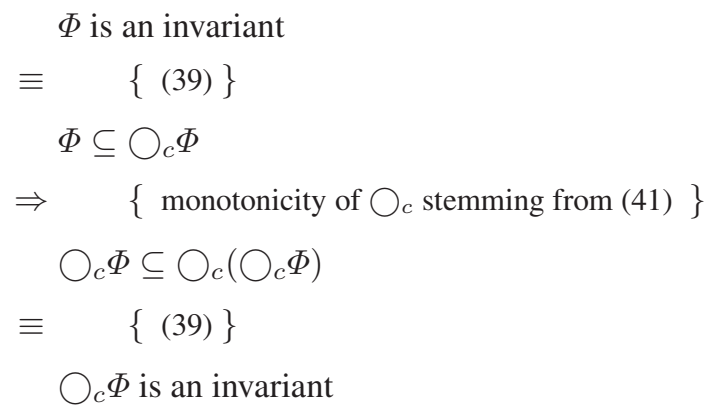

The whole construction of a modal logic relative to a coalgebra $c$, which is the basis of assertion reasoning in coalgebra theory, can be pursued along similar lines. Consider, for example, the definition of $\square P$, the henceforth $P$ operator of [11, Def. 4.2.8]:

$$
(\square P) x \stackrel{\text { def }}{=}\langle\exists Q: Q \text { is invariant : } Q \subseteq P \wedge(Q x)\rangle
$$

Converting predicates $P$ and $Q$ to coreflexives $\Phi$ and $\Psi$, respectively, and making explicit the supremum implicit in the existential quantification one gets,

$$
\begin{aligned}
\square \Phi= & \left\langle\bigcup \Psi: \Psi \subseteq \bigcirc_{c} \Psi: \Psi \subseteq \Phi\right\rangle \\
= & \{\text { trading [4] }\} \\
& \left\langle\bigcup \Psi:: \Psi \subseteq \bigcirc_{c} \Psi \wedge \Psi \subseteq \Phi\right\rangle \\
= & \quad\{\cap \text {-universal }\} \\
& \left\langle\bigcup \Psi:: \Psi \subseteq \bigcirc_{c} \Psi \cap \Phi\right\rangle \\
= & \{\cap \text { of coreflexives is composition 442 }\} \\
& \left\langle\bigcup \Psi:: \Psi \subseteq \Phi \cdot \bigcirc_{c} \Psi\right\rangle
\end{aligned}
$$

which leads to a greatest (post)fixpoint definition:

$$
\square \Phi=\left\langle\nu \Psi:: \Phi \cdot \bigcirc_{c} \Psi\right\rangle
$$

We end this section by showing how the PF-transform (and in particular the replacement of intersection of coreflexives by composition (42) together with the fixpoint calculus [3] speed up derivation of laws in such a logic. The law we have chosen to calculate is Lemma 4.2.9(ii) of [11]: $\square \Phi \subseteq \square \square \Phi$. We drop subscript $c$ of $\bigcirc_{c}$ (for economy of notation) and calculate:

$$
\begin{aligned}
\square \Phi & \subseteq \square \square \Phi \\
\equiv \quad & \{43\}
\end{aligned}
$$




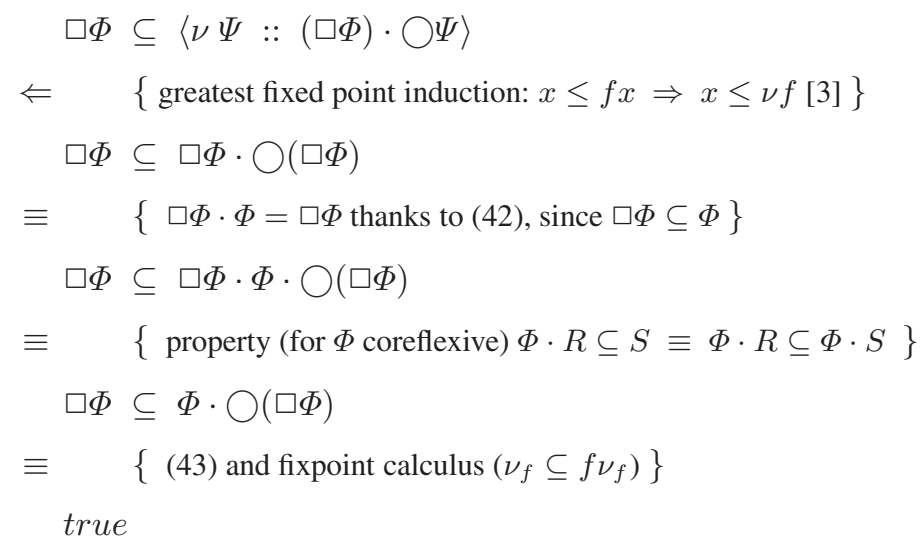

\section{Epilogue}

Invariants are constraints on the carrier of coalgebras which restrict their behavior in some desirable way but whose maintenance entails some kind of proof obligation discharge. An approach is put forward in this paper for reasoning about coalgebraic invariants which is both compositional and calculational: compositional because it is based on rules which break the complexity of such proof obligations across the structures involved; calculational because such rules are derived thanks to an algebra of invariants regarded as coreflexive bisimulations, which is what invariants are once encoded in the language of binary relations. Such calculational capabilities arise, in turn, from encoding bisimulations as instances of Reynolds relation on functions. In this process, functors which capture coalgebras' dynamics are generalized to relators and the objects of the underlying category are generalized to predicates.

The main contribution of the paper is the explicit adoption of such a constructive, calculational style in approaching the problem. Both [216] already suggest a relational/relator-based approach to bisimulation, [6] actually generalizing from coalgebras to dialgebras. However, no relationship is established with the algebra of Reynolds relation on functions which, in close association with Reynolds abstraction theorem, naturally leads to a category (Pred) whose objects are predicates (invariants).

In a wider context, the explicit adoption of such a category has potential to support a constructive discipline of extended static checking (ESC) in a coalgebraic view of computer systems, but surely there is much work to be done before this becomes of practical use. On the theory side, the authors would like to investigate a possible connection between the "predicates as objects" approach and Frege structures [10] 12. Quoting this reference:

A Frege structure is a lambda structure $\mathcal{F}$ on the set $A$ together with a designated subset of $A$ whose elements are called propositions (...) the propositional connectives are required to yield propositions as values only when they operate on propositions as arguments.

This is regarded as an interesting topic for future research.

\footnotetext{
${ }^{12}$ We thank Peter Dybjer for pointing out this possibility.
} 


\section{References}

1. Aczel, P., Mendler, N.: A final coalgebra theorem. In: Category Theory and Computer Science, London, UK, pp. 357-365. Springer, Heidelberg (1989)

2. Backhouse, K., Backhouse, R.C.: Safety of abstract interpretations for free, via logical relations and Galois connections. SCP 15(1-2), 153-196 (2004)

3. Backhouse, R.: Galois connections and fixed point calculus. In: Crole, R., Backhouse, R., Gibbons, J. (eds.) Algebraic and Coalgebraic Methods in the Mathematics of Program Constuction. LNCS, vol. 2297, pp. 89-148. Springer, Heidelberg (2002)

4. Backhouse, R., Michaelis, D.: Exercises in quantifier manipulation. In: Uustalu, T. (ed.) MPC 2006. LNCS, vol. 4014, pp. 70-81. Springer, Heidelberg (2006)

5. Backhouse, R.C., de Bruin, P., Hoogendijk, P., Malcolm, G., Voermans, T.S., van der Woude, J.: Polynomial relators. In: AMAST 1991, pp. 303-362. Springer, Heidelberg (1992)

6. Backhouse, R.C., Hoogendijk, P.F.: Final dialgebras: From categories to allegories. Informatique Theorique et Applications 33(4/5), 401-426 (1999)

7. Bird, R., de Moor, O.: Algebra of Programming. Hoare, C.A.R.(series ed.). Series in Computer Science. Prentice-Hall International, Englewood Cliffs (1997), http: / / www .phptr.com/ptrbooks/ptr_013507245x.html

8. Carboni, A., Kelly, G., Wood, R.: A 2-categorical approach to change of base and geometric morphisms I. Technical Report 90-1, Dept. of Pure Maths, Univ. Sydney (1990)

9. Freyd, P.J., Ščedrov, A.: Categories, Allegories. Mathematical Library, vol. 39. NorthHolland, Amsterdam (1990)

10. Hatcher, W.S.: Review: Peter Aczel. Frege structures and the notions of proposition, truth and set. The Journal of Symbolic Logic 51(1), 244-246 (1986)

11. Jacobs, B.: Introduction to Coalgebra. Towards Mathematics of States and Observations. Draft Copy. Institute for Computing and Information Sciences, Radboud University Nijmegen, P.O. Box 9010, 6500 GL Nijmegen, The Netherlands

12. Jones, C.B.: Systematic Software Development Using VDM. Prentice-Hall Int., Englewood Cliffs (1986)

13. Kock, A.: Strong functors and monoidal monads. Archiv für Mathematik 23, 113-120 (1972)

14. Oliveira, J.N.: Invariants as coreflexive bisimulations — in a coalgebraic setting, Presentation at the IFIP WG 2.1 \#62 Meeting Namur (December 2006)

15. Oliveira, J.N.: Pointfree foundations for (generic) lossless decomposition (submitted, 2007)

16. Oliveira, J.N.: Theory and applications of the PF-transform, Tutorial at LerNET 2008, Piriàpolis, Uruguay (slides available from the author's website) (February 2008)

17. Oliveira, J.N., Rodrigues, C.J.: Pointfree factorization of operation refinement. In: Misra, J., Nipkow, T., Sekerinski, E. (eds.) FM 2006. LNCS, vol. 4085, pp. 236-251. Springer, Heidelberg (2006)

18. Park, D.: Concurrency and automata on infinite sequences. LNCS, vol. 104, pp. 561-572. Springer, Heidelberg (1981)

19. Pratt, V.: Origins of the calculus of binary relations. In: Proc. of the 7th Annual IEEE Symp. on Logic in Computer Science, Santa Cruz, CA, pp. 248-254. IEEE Computer Society Press, Los Alamitos (1992)

20. Reynolds, J.C.: Types, abstraction and parametric polymorphism. Information Processing 83, 513-523 (1983)

21. Rutten, J.J.M.M.: Relators and metric bisimulations. ENTCS 11, 1-7 (1998)

22. Rutten, J.J.M.M.: Coalgebraic foundations of linear systems. In: Mossakowski, T., Montanari, U., Haveraaen, M. (eds.) CALCO 2007. LNCS, vol. 4624, pp. 425-446. Springer, Heidelberg (2007) 
23. Tarski, A., Givant, S.: A Formalization of Set Theory without Variables. American Mathematical Society, vol. 41. AMS Colloquium Publications, Providence (1987)

24. Vaccari, M.: Calculational derivation of circuits. PhD thesis, Univ. S. Milano (1998)

25. Wadler, P.L.: Theorems for free! In: 4th Int. Symp. on FPLCA, London, September 1989. ACM Press, New York (1989) 